World Health Organization (1992) The ICD-10 Classification of Mental and Behaviour Disorders-Clinical descriptions and diagnostic guidelines. Geneva: WHO.

Prince of Wales Hospital

Sing LeB

Shatin, Hong Kong

\section{Rhabdomyolysis due to mania}

SIR: Rhabdomyolysis commonly occurs in response to trauma, limb ischaemia, severe exertion or prolonged stasis. It has, however, been associated with wolf attacks, conga-drumming, karate, mechanical bull-riding and deep knee bends (Frucht, 1994). Following injury skeletal muscle enters an accelerated catabolic state releasing myoglobin which is really excreted and precipitates in the renal tubules leading to acute renal failure in up to one-third of cases. Classically the condition presents with myalgia, muscle swelling and tenderness with deep red brown urine. However, incipient deterioration of renal function, haematuria or proteinuria may be the only features. The serum creatinine kinase rises by up to a magnitude of 100 with the $\mathrm{MM}$ isoenzyme representing greater than $97 \%$ of the total. We report a case of rhabdomyolysis associated with acute mania.

A 67-year-old man presented with a one-week history of profound overactivity, agitation and insomnia. Six months earlier he had been admitted briefly with a similar episode which had been treated successfully with a short course of lorazepam. There was no history of drug abuse and no family history of psychiatric illness. On examination he was profoundly overactive, pacing the room throughout the interview becoming progressively agitated and threatening. He was markedly sexually disinhibited making lurid comments to female staff. Florid pressure of speech with flight of ideas was present although he did not appear deluded or to be hallucinating. Physical examination was normal.

Lorazepam $4 \mathrm{mg}$ per day was commenced and he remained freely mobile on the ward refusing all but minimal quantities of water. His mood settled on the lorazepam but four days following admission he complained of general malaise, worsening bilateral calf muscle pain and darkening of his urine. On examination he was apyrexial, moderately dehydrated with a uraemic foetor. There was no evidence of tissue injury although he had extremely tender calves and forearms. Urinalysis revealed $3+$ protein with no blood. Biochemistry demonstrated a deterioration in renal function with a serum urea of $20.4 \mathrm{mmol} / \mathrm{h}$ and creatinine of $300 \mathrm{mmol} / \mathrm{h}$. Creatinine kinase was grossly elevated at $7234 \mathrm{iu} / 1$ (nr 25-195 iu/l) with MM fraction of $98 \%$. He had myoglobinuria $(>100000 \mathrm{iu} / \mathrm{l})$. He was treated with simple analgesics and intravenous fluids. Urinalysis was negative within five days and the creatinine kinase concentration returned to normal over one week.

To our knowledge, this is the first reported case of rhabdomyolysis due to excessive exertion and dehydration secondary to an acute manic episode though we suspect that mild cases may remain undetected. Patients with psychiatric illness are particularly susceptible to rhabdomyolysis secondary to alcohol abuse of neuroleptic medication but only two other cases of rhabdomyolysis resulting directly from psychotic disorders have been reported. (Coryell et al, 1978; Frankel \& Prassad, 1989). In the first a patient with a known psychotic illness developed rahdomyolysis after assuming a catatonic position for an hour. In the second, a chronic schizophrenic required dialysis following four hours continual jumping up and down during an acute psychotic episode. Rhabdomyolysis is a serious and potentially fatal complication of psychosis and should be considered in all cases of overactivity or catatonia.

Coryell, W., Norby, L. H. \& Cohen, L. H. (1978) Psychosis induced by rhabdomyolysis. Lancet, 8085, 381-382.

Frankel, A. S. \& Prassad, R. B. (1989) Rhabdomyolysis, renal failure and schizophrenia. Psychiatry Journal of the University of Ottawa, 141, 296-297.

Frucht, M. (1994) Challenge, 110 deep knee bends; reward, rhabdomyolysis. New England Journal of Medicine, 330, 1620 1621 .

S.M. MANCHIP S.J. HUREL

University of Newcastle Medical School

Newcastle Upon Tyne NE2 $4 \mathrm{HH}$

Cardiovascular and autonomic function in Down syndrome-prescribing implications

SIR: Although modern literature on Down syndrome routinely lists such problems as hypothyroidism, sleep apnoea and atlanto-axial instability, it rarely mentions the evidence of autonomic dysfunction in Down syndrome, which should affect medical prescribing. There has been evidence available for some time, with "the not infrequently fatal idiosyncrasy" of Down syndrome to atropinerelated drugs commented on in 1957 by McKusick and with later experimental evidence of hyperreactivity to atropine (Mir \& Cumming, 1971). Since then there has been further evidence of an abnormal sympathetic nervous system in terms of enzyme levels and response to noradrenaline (Lake 